\title{
Selfadjoint Extensions for the Elasticity System in Shape Optimization
}

\author{
by \\ Serguei A. NAZAROV and Jan SOKOŁOWSKI \\ Presented by Jan KISYŃSKI
}

Summary. Two approaches are proposed to modelling of topological variations in elastic solids. The first approach is based on the theory of selfadjoint extensions of differential operators. In the second approach function spaces with separated asymptotics and point asymptotic conditions are introduced, and a variational formulation is established. For both approaches, accuracy estimates are derived.

1. Introduction. It seems that in the literature on shape optimization there is a lack of general techniques or mathematical framework that could be applied in the process of optimization of an arbitrary shape functional (SF) for simultaneous boundary and topology variations. From the numerical point of view there are, however, some efficient methods including the homogenization technique [1], [6] or the level set method [2] which can be used, in particular, for topology optimization.

In [24], [20] (see also [8], [9], [25]) the so-called topological derivative (TD) of an arbitrary SF is introduced. TD usually determines if the value $J(\Omega)$ of a given SF can be improved or not when a small hole is introduced in the geometrical domain with centre at a given point $\boldsymbol{x} \in \Omega$. In the same setting the influence of a small inclusion in the domain, with different material properties, on the value of the SF can be considered. Such a change of the topology of the geometrical domain implies a singular perturbation of the domain. In asymptotic analysis some integral attributes of singu-

2000 Mathematics Subject Classification: Primary 35B40, 35C20; Secondary 49Q10, $74 \mathrm{P} 15$.

Key words and phrases: elasticity boundary value problem, singular perturbation of geometrical domain, selfadjoint extension, shape optimization, topological derivative of shape functional. 
lar perturbations are determined [19], for example polarization matrices. In the present paper the boundary topology variations are considered for a mixed boundary value problem for elastic solids. Singular perturbations of the boundary value problem by a change of boundary conditions on a small set are defined by small $\operatorname{arcs} \gamma_{h}^{1}, \ldots, \gamma_{h}^{J}$ of length $O(h)$ on the boundary $\partial \Omega$.

We propose two efficient approaches to modelling of topological variations. The first approach is developed in the framework of selfadjoint extensions of differential operators, the second uses function spaces with separated asymptotics. In both cases, the main idea consists in modelling small defects or inhomogeneities by concentrated actions, the so-called potentials of zeroradii. In this way the solution $u(\varepsilon, h)$ with singular behaviour for $\varepsilon \rightarrow 0+$ is replaced by a function with singularities at the centres $P^{1}, \ldots, P^{J}$ of the defects. The modern analysis of elliptic boundary value problems in nonsmooth domains yields a fairly complete theory of singular solutions and provides techniques of derivation of error estimates for asymptotic approximations. We can use the known results in this field for solution of shape and topology optimization problems in reverse order. First, the localization and integral atributes of openings are determined, followed by appropriate changes of the topology of geometrical domains. The proposed two different approaches to topology optimization have some properties that may be useful for further applications, in particular in numerical methods. The first approach deals with selfadjoint operators, so can be readily extended to evolution boundary value problems. The second approach, based on generalized Green formulae, results in variational formulations with the solution given by a stationary point of an auxiliary functional close in its form to the energy functional.

General results on modelling of shape functionals and asymptotic analysis can be found in [20]. The results presented in this paper can be obtained by an application of that technique.

2. Problem formulation. Let us consider deformations of a plane heterogeneous anisotropic elastic body $\Omega \subset \mathbb{R}^{2}$ clamped or supported on small parts of the boundary $\Gamma=\partial \Omega$ in the form of closed connected curves $\gamma_{h}^{1}, \ldots, \gamma_{h}^{J}$. Instead of tensor notation, we make use of matrix notation which we describe briefly. The constitutive relations in elasticity theory are written with elastic fields in the form of vectors. First, two matrices are introduced:

$$
D(x)^{\top}=\left[\begin{array}{ccc}
x_{1} & 0 & \alpha x_{2} \\
0 & x_{2} & \alpha x_{1}
\end{array}\right], \quad d(x)^{\top}=\left[\begin{array}{ccc}
1 & 0 & -\alpha x_{2} \\
0 & 1 & \alpha x_{1}
\end{array}\right],
$$

where $\alpha=2^{-1 / 2}$ is the normalizing coefficient, and $\top$ stands for transposition. The first matrix is used to define the column of strains from the 
displacement column $u=\left(u_{1}, u_{2}\right)^{\top}$,

$$
\varepsilon(u):=\left(\varepsilon_{11}(u), \varepsilon_{22}(u), \alpha^{-1} \varepsilon_{12}(u)\right)^{\top}=D(\nabla) u .
$$

Here $\nabla=\left(\partial / \partial x_{1}, \partial / \partial x_{2}\right)^{\top}$ is the gradient, and $\varepsilon_{j k}(u)$ are the Cartesian components of the strain tensor (the factors $\alpha$ in (1) and (2) make the norms of the vector and tensor of strains equal). The second matrix generates the rigid motions $d(x)^{\top} b$ of the body $\Omega$ for any column $b \in \mathbb{R}^{3}$. Hooke's law

$$
\sigma(u ; x)=A(x) \varepsilon(u ; x)
$$

expresses the column of stresses as a function of the strains (2), and includes a symmetric and positive definite $(3 \times 3)$-matrix function $A$, which is supposed to be a smooth function of $x$. In view of (1)-(3), the equilibrium equations and the boundary conditions of traction free type are given as follows:

$$
\begin{array}{ll}
L(x, \nabla) u(h, x):=D(-\nabla)^{\top} A(x) D(\nabla) u(h, x)=f(x), & x \in \Omega, \\
B(x, \nabla) u(h, x):=D(n(x))^{\top} A(x) D(\nabla) u(h, x)=0, & x \in \Sigma_{h} .
\end{array}
$$

Here, $n=\left(n_{1}, n_{2}\right)^{\top}$ is the unit external normal vector to the contour $\Gamma$, which is supposed to be sufficiently smooth for simplicity. In (5), $\Sigma_{h}=$ $\Gamma \backslash\left\{\gamma_{h}^{1} \cup \cdots \cup \gamma_{h}^{I}\right\}$, and $\gamma_{h}^{j}$ are arcs of length $h l_{j}$, with centres $P^{j} \in \partial \Omega$, where $h \in\left(0, h_{0}\right]$ is a small parameter and $l_{1}, \ldots, l_{J}$ are fixed constants. The elastic body is either clamped on the sets $\gamma_{h}^{j}$ or in contact with a rigid foundation, so the linearized contact conditions are possibly prescribed on the $\operatorname{arcs} \gamma_{h}^{I+1}, \ldots, \gamma_{h}^{J}$ with $I \leq J$,

$$
\begin{array}{rlrl}
u(h, x) & =0, & x \in \gamma_{h}^{1} \cup \cdots \cup \gamma_{h}^{I}, \\
u_{n}(h, x):=n(x)^{\top} u(h, x) & =0, & x \in \gamma_{h}^{I+1} \cup \cdots \cup \gamma_{h}^{J}, \\
s(x)^{\top} D(n(x))^{\top} A(x) D(\nabla) u(h, x) & =0, & & x \in \gamma_{h}^{I+1} \cup \cdots \cup \gamma_{h}^{J},
\end{array}
$$

with tangent vector $s=\left(-n_{2}, n_{1}\right)^{\top}$. We point out that a proper formulation of the contact conditions requires the application of the Signorini conditions instead of (7). The simpler condition (7) does not imply the unilateral conditions $n^{\top} D(n)^{\top} A D(\nabla) u \leq 0$ on $\gamma_{h}^{I+1}, \ldots, \gamma_{h}^{J}$ required in the variational formulation of frictionless contact of an elastic body with a rigid foundation. Such a unilateral relation means that the reaction of the foundation is non-negative at each point of the specific contact zone. Nevertheless, there are particular situations such that the solution of the Signorini problem formulated in a proper way coincides with the solution of problem (4)-(8) (cf. [4], and see Example 1). The cases of $I=J$ and $I=0$ are not excluded from our modelling. 
3. Korn inequality. For $I>0$ the Dirichlet condition provides the Korn inequality

$$
\left\|u ; H^{1}(\Omega)\right\| \leq K(h)\left\|D(\nabla) u ; L_{2}(\Omega)\right\|,
$$

but the dependence of $K(h)$ on $h$ is not known in general. We indicate geometrical conditions which allow us to evaluate the order of $K(h)$ as $h \rightarrow+0$. Such an estimate is used to justify the proposed models of problem (4)-(6), (8), singularly perturbed due to the presence of a small parameter $h$ in the boundary conditions.

We say that a column $a \in \mathbb{R}^{2}$ is admissible at a point $P^{j}$ if $a \in \mathbb{R}^{2}$ for $j=1, \ldots, I$ (for clamped $\gamma_{h}^{j}$ ) or $a$ is parallel to the normal $n\left(P^{j}\right)$ for $j=I+1, \ldots, J$ (in the case of contact along $\gamma_{h}^{j}$ ). In what follows it is always assumed that the following condition is satisfied.

(H1) The linear hull of the columns $d\left(P^{j}\right)^{\top} a^{j} \in \mathbb{R}^{3}$, where $j=1, \ldots, J$ with $a^{j} \in \mathbb{R}^{2}$ admissible at $P^{j}$, is the whole space $\mathbb{R}^{3}$.

Proposition 1. Under condition (H1), for any field $u \in H^{1}(\Omega)^{2}$ satisfying the Dirichlet conditions (6)-(7), the Korn inequality holds with

$$
K(h) \leq c|\ln h| .
$$

The estimate is asymptotically exact, and the constant $c$ is independent of $u$ and $h \in\left(0, h_{0}\right]$ with $h_{0}<1$.

ExAmple. (a) If $I \geq 2$ or $I=1, J \geq 2$, then condition (H1) is satisfied.

(b) If $I=0$, then for any distribution of contact regions $\gamma_{h}^{1}, \ldots, \gamma_{h}^{J}$ on the boundary of the disk $\omega=\{x:|x|<R\}$ the Korn inequality (9) is not valid.

(c) If $I=J=1$, then condition (H1) is violated. Nevertheless, the Korn inequality (9) is valid, but $K(h)$ becomes of order $h^{-1}$ (cf. [23]), and thus it does not satisfy estimate (10).

(d) If $I=1, J=2, P^{1}=(0, R), P^{2}=(0,-R)$ and $f(x)=(0,-c)$, $c>0$, then the solution of problem (4)-(6), (8) in the disk coincides with the solution of the Signorini problem in the same disk with the Dirichlet condition on $\gamma_{h}^{1}$ and the unilateral condition on $\gamma_{h}^{2}$.

\section{Modelling of a singularly perturbed boundary value problem.}

Since there are changes of the boundary conditions at the tips of small arcs, the boundary value problem (4)-(6), (8) can be viewed as singularly perturbed. The theory established in [14], [10] and others yields a complete asymptotic expansion of the solution $u(h, x)$ with accuracy $O\left(h^{N}\right)$, where $N$ can be a priori fixed arbitrarily large. In the same way as in [20], such asymptotic formulae are applicable to the analysis of shape functionals by 
means of topological derivatives. We consider the functional

$$
\mathcal{F}(u ; h)=\int_{\Omega} J(x ; u(h, x)) d x .
$$

In addition, the asymptotic structures for specific problems with a logarithmic growth of fundamental solutions turn out to be quite complex, and therefore of limited practical interest for analysis of the functional (11). The main feature of the asymptotic analysis, besides the presence of boundary layers near the $\operatorname{arcs} \gamma_{h}^{1}, \ldots, \gamma_{h}^{J}$, is the form of asymptotic terms which are rational functions of the large parameter $|\ln h|$. This phenomenon was discovered by A. M. Il'in for the scalar problem in [11] (see also [10] and [14]).

A simplification caused by regarding asymptotics with respect to $|\ln h|^{-1}$ is not sufficient to perform the analysis, since the leading terms of the asymptotics do not reflect the distribution of contact regions and do not exhibit interactions between the regions.

We propose an approach, based on modelling problem (4)-(6), (8) by means of auxiliary boundary value problems with the boundary conditions of traction free type on the punctured contour $\partial \Omega \backslash\left\{P^{1}, \ldots, P^{J}\right\}$ and with singularities of prescribed class at the points $P^{1}, \ldots, P^{J}$. Such singularities are obtained by application of forces concentrated at those points, and therefore, imitate the reaction of the elastic body at the obstacles $\gamma_{h}^{1}, \ldots, \gamma_{h}^{J}$. Thus, in this setting, the models take into account the interaction of the rigid foundations through the elastic body. On the other hand, the proposed singularities are not in the energy class $H^{1}(\Omega)^{2}$, but the resulting singular solutions are still in the space $L_{q}(\Omega)$, for $q \geq 1$. The main profit from our point of view is the possibility to obtain, by means of singular solutions, an asymptotically exact approximation of the functional (11), under the condition that for some $q \in[1, \infty)$ and for any $u, v \in L_{q}(\Omega)^{2}$ the following inequality is valid:

$$
\begin{aligned}
\mid \mathcal{F}(u ; h)- & \mathcal{F}(v ; h) \mid \\
& \leq c_{\mathcal{F}}\left\|u-v ; L_{q}(\Omega)\right\|\left(\left\|u ; L_{q}(\Omega)\right\|^{q-1}+\left\|v ; L_{q}(\Omega)\right\|^{q-1}\right)
\end{aligned}
$$

with the constant $c_{\mathcal{F}}$ independent of $h \in\left(0, h_{0}\right]$ and $u, v$.

Modelling defects in media by an application of extensions of differential operators which give rise to singular solutions originates from [7] and is developed e.g. in [22], [16], [19], [18] for problems of mathematical physics and general elliptic systems. In particular, three-dimensional contact problems are as well investigated in this direction in [4].

There are two possibilities for realization of such ideas. First of all, the operator $L(x, \nabla)$ in $(4)$, considered as an unbounded operator in the space $L_{2}(\Omega)^{2}$, is restricted to a smaller domain of definition compared to its natural domain $H^{1}(\Omega)^{2}$. In this way the domain of the adjoint operator becomes 
wider, and a proper choice of extension parameters can be used to select an appropriate selfadjoint operator. The selfadjoint operator $\mathbf{L}$ with properly selected extension parameters asymptotically inherits the attributes, in other words intrinsic characteristics, of the singularly perturbed problem such as the energy functional and the spectrum (see [18], [20]). In addition, the classical semigroup theory can be used to construct solutions for the evolution problems associated with the selfadjoint operator. On the other hand, the domain $\mathcal{D}(\mathbf{L})$ of the selected selfadjoint extension depends on the large parameter $|\ln h|$, which could be inconvenient for numerical methods when applied to shape optimization or shape inverse problems (see [9], [24], [25], [8]). This difficulty can be avoided by application of a slightly different technique, including a space with separated asymptotics (see e.g. [19]). Roughly speaking, the boundary value problem is defined and solved in a larger class, compared even with $\mathcal{D}(\mathbf{L})$. The uniqueness of solutions is obtained by conditions at the points $P^{1}, \ldots, P^{J}$. Such asymptotic point conditions at $P^{1}, \ldots, P^{J}$ serve as an additional relation between the coefficients of prescribed asymptotic expansions. In particular, by construction, exactly the same solutions are obtained by the first and the second approach.

5. Modelling with selfadjoint extensions. The unbounded operator $\mathcal{L}$ in $L_{2}(\Omega)^{2}$ defined by the differential expression $L(x, \nabla)$ with domain

$$
\begin{aligned}
\mathcal{D}(\mathcal{L}) & =\left\{v \in H^{2}(\Omega)^{2}: B(x, \nabla) v=0 \text { on } \partial \Omega,\right. \\
& \left.v\left(P^{1}\right)=\cdots=v\left(P^{I}\right)=0 \in \mathbb{R}^{2}, v_{n}\left(P^{I+1}\right)=\cdots=v_{n}\left(P^{J}\right)=0\right\}
\end{aligned}
$$

is closed and symmetric, but the adjoint $\mathcal{L}^{*}$ has a larger domain:

$$
\mathcal{D}\left(\mathcal{L}^{*}\right)=\left\{v \in \mathfrak{D}: B(x, \nabla) v=0 \text { on } \partial \Omega \backslash\left\{P^{1}, \ldots, P^{J}\right\}\right\},
$$

where

$$
\begin{aligned}
& \mathfrak{D}=\left\{v(x)=\widetilde{v}(x)+\sum_{i=1}^{I} \chi_{i}(x)\left[b^{i}+T^{i}\left(x-P^{i}\right) a^{i}\right]\right. \\
& \quad+\sum_{j=I+1}^{J} \chi_{j}(x)\left[n\left(P^{j}\right) b_{j}+T^{j}\left(x-P^{j}\right) a_{j} n\left(P^{j}\right)\right]: \\
& \widetilde{v} \in H^{2}(\Omega)^{2}, \widetilde{v}^{1}\left(P^{1}\right)=\cdots=\widetilde{v}^{I}\left(P^{I}\right)=0, \widetilde{v}_{n}^{I+1}\left(P^{I+1}\right)=\cdots=\widetilde{v}_{n}^{J}\left(P^{J}\right)=0, \\
& \left.a^{i}, b^{i} \in \mathbb{R}^{2}, a_{j}, b_{j} \in \mathbb{R}\right\} .
\end{aligned}
$$

Here $\chi_{1}, \ldots, \chi_{J}$ are cutoff functions with mutually disjoint supports, equal to one in neighbourhoods of $P^{1}, \ldots, P^{J}$, respectively, and $T^{j}=\left(T^{j 1}, T^{j 2}\right)$ is the Poisson kernel, i.e., a $(2 \times 2)$-matrix function, where each column $T^{j k}$, $k=1,2$, is a solution of the elasticity boundary value problem in the half- 
plane $\left\{x: n\left(P^{j}\right)^{\top} x>0\right\}$ under unit force concentrated at the point $P^{j}$ and directed in the positive direction of the $x_{k}$-axis (such solutions $T^{j k}$ are linear combinations of the Boussinesq-Cerruti solutions [21]). The following representation is well known:

$$
T^{j}(x)=-\mathcal{T}^{j 0} \ln |x|+\mathcal{T}^{j 1}\left(|x|^{-1} x\right),
$$

where $\mathcal{T}^{j 1}$ is a smooth matrix function on the semisphere and $\mathcal{T}^{j 0}$ is a constant $(2 \times 2)$-matrix, symmetric and positive definite.

By comparison of formulae (13) and (14) we can see that the defect of the operator $\mathcal{L}$ is $(N: N)$, where $N=2 I+(J-I)=J+I$. The coefficients $b_{1}^{i}, b_{2}^{i}$ and $b_{j}$ from (14) form a column $\mathbf{b} \in \mathbb{R}^{N}$, the remaining coefficients form a column a.

Lemma 1. Let $\mathbf{S}$ be a symmetric $(N \times N)$-matrix. The restriction $\mathbf{L}$ of the operator $\mathcal{L}^{*}$ to the linear subspace

$$
\mathcal{D}(\mathbf{L})=\left\{v \in \mathcal{D}\left(\mathbf{L}^{*}\right): \mathbf{b}=\mathbf{S a}\right\}
$$

is a selfadjoint operator in $L_{2}(\Omega)^{2}$. If the matrix $\mathbf{S}$ is not singular, then under condition $(\mathrm{H} 1)$ the equation

$$
\mathbf{L v}=f
$$

admits a unique solution for each $f \in L_{2}(\Omega)^{2}$.

A proper choice of parameters of the selfadjoint extension, i.e., a selection of the matrix $\mathbf{S}$ is performed in Section 7 in such a way that the solution $\mathbf{v}$ of equation (17) becomes an approximation of the solution to problem (4)-(6), (8).

6. Modelling in spaces with separated asymptotics. The linear space (14) with the norm

$$
\|v ; \mathfrak{D}\|=\left(\left\|\widetilde{v} ; H^{2}(\Omega)\right\|^{2}+\left\|\mathbf{a} ; \mathbb{R}^{N}\right\|^{2}+\left\|\mathbf{b} ; \mathbb{R}^{N}\right\|^{2}\right)^{1 / 2}
$$

becomes a Hilbert space. We introduce two projection operators $\pi^{ \pm}$: $\mathfrak{D} \rightarrow \mathbb{R}^{N}$ by

$$
\pi^{-} v=\mathbf{a}, \quad \pi^{+} v=\mathbf{b} .
$$

Let us consider the boundary value problem of linear elasticity with the asymptotic conditions at the points $P^{1}, \ldots, P^{J}$,

$$
\begin{array}{llrl}
L(x, \nabla) \mathfrak{v}(x) & =f(x), & & x \in \Omega, \\
B(x, \nabla) \mathfrak{v}(x)=0, & & x \in \partial \Omega \backslash\left\{P^{1}, \ldots, P^{J}\right\}, \\
S \pi^{-} \mathfrak{v}-\pi^{+} \mathfrak{v}=0 \in \mathbb{R}^{N} . & &
\end{array}
$$

It is easy to see that for $S=\mathbf{S}$ the solutions $\mathbf{v} \in \mathcal{D}(\mathbf{L})$ of (17) and $\mathfrak{v} \in \mathfrak{D}$ of (18) coincide. 
Proposition 2. (i) For $\mathfrak{v}, \mathfrak{u} \in \mathfrak{D}$ the following generalized Green formula is valid:

$$
\begin{aligned}
(L \mathfrak{v}, \mathfrak{u})_{\Omega}+(B \mathfrak{v}, \mathfrak{u})_{\partial \Omega} & +\left\langle S \pi^{-} \mathfrak{v}-\pi^{+} \mathfrak{v}, \pi^{-} \mathfrak{u}\right\rangle \\
& =(\mathfrak{v}, L \mathfrak{u})_{\Omega}+(\mathfrak{v}, B \mathfrak{u})_{\partial \Omega}+\left\langle\pi^{-} \mathfrak{v}, S \pi^{-} \mathfrak{u}-\pi^{+} \mathfrak{u}\right\rangle
\end{aligned}
$$

where $(\cdot, \cdot)_{\Xi}$ and $\langle\cdot, \cdot\rangle$ are the scalar products in the spaces $L_{2}(\Xi)^{2}$ and $\mathbb{R}^{N}$, respectively.

(ii) The function $\mathfrak{v} \in \mathfrak{D}$ is a solution to problem (18) if and only if it is a stationary point of the functional

$$
\mathfrak{E}(\mathfrak{v})=\frac{1}{2}(L \mathfrak{v}, \mathfrak{v})_{\Omega}+\frac{1}{2}(B \mathfrak{v}, \mathfrak{v})_{\partial \Omega}+\frac{1}{2}\left\langle S \pi^{-} \mathfrak{v}-\pi^{+} \mathfrak{v}, \pi^{-} \mathfrak{v}\right\rangle-(f, \mathfrak{v})_{\Omega} .
$$

If $\operatorname{det} S \neq 0$ and condition (H1) is satisfied, then the stationary point of the functional (20) is uniquely determined.

The symmetric generalized Green formula shows that the boundary value problem is formally selfadjoint.

The second assertion in Proposition 2 furnishes the variational formulation of problem (18) over the Hilbert space $\mathfrak{D}$, and shows the uniqueness of solutions under the same conditions as in the case of equation (17).

7. How to determine the model parameters. The solution $\mathbf{v}=\mathfrak{v}$ of equation (17) or of problem (18) satisfies system (4) and boundary conditions (5), however, in general, fails to satisfy the boundary conditions (6) and $(7),(8)$. In order to construct an approximation for the solution $u(h, x)$ in the vicinity of the points $P^{1}, \ldots, P^{J}$, the method of matched asymptotic expansions is applied (see [10], [13], and cf. [14], [19]). Thus, selecting $\mathbf{v}=\mathfrak{v}$ for the outer asymptotic expansion, we construct the inner expansions $w^{j}\left(\xi^{j}\right)$, employing the fast variables $\xi^{j}=h^{-1}\left(x-P^{j}\right)$. Formally, we change the variables and pass to the limit as $h \rightarrow+0$. In the limit $h \rightarrow+0$ the dilatation of coordinates implies the rectifying of the boundary, freezing of the coefficients at the point $P^{j}$, and vanishing of the volume forces in the equilibrium equations. The procedure leads to the boundary value problem for $w^{j}$ which consists of the homogeneous elasticity system

$$
D\left(-\nabla_{\xi}\right)^{\top} A\left(P^{j}\right) D\left(\nabla_{\xi}\right) w^{j}(\xi)=0, \quad \xi \in \mathbb{R}_{j}^{2},
$$

the boundary conditions of traction free type

$$
D\left(n^{j}\right)^{\top} A\left(P^{j}\right) D\left(\nabla_{\xi}\right) w^{j}(\xi)=0, \quad \xi \in \partial \mathbb{R}_{j}^{2},|\xi|>l_{j} / 2,
$$

the Dirichlet conditions for $j=1, \ldots, I$,

$$
w^{j}(\xi)=0, \quad \xi \in \partial \mathbb{R}_{j}^{2}, \quad|\xi|<l_{j} / 2,
$$

and the linearized contact conditions for $j=I+1, \ldots, J$, 


$$
\begin{aligned}
&\left(n^{j}\right)^{\top} w^{j}(\xi)=0, \quad\left(s^{j}\right)^{\top} D\left(n^{j}\right)^{\top} A\left(P^{j}\right) D\left(\nabla_{\xi}\right) w^{j}(\xi)=0, \\
& \xi \in \partial \mathbb{R}_{j}^{2}, \quad|\xi|<l_{j} / 2 .
\end{aligned}
$$

Here $n^{j}=n^{j}\left(P^{j}\right)$ and $s^{j}=s^{j}\left(P^{j}\right)$ are normal and tangential vectors on $\partial \Omega$, respectively, evaluated at $P^{j} ; \mathbb{R}_{j}^{2}$ is the half-plane $\left\{\xi \in \mathbb{R}^{2}: \xi^{\top} n^{j}<0\right\}$.

Since we are going to glue $w^{j}$ with the singular solution $\mathbf{v}=\mathfrak{v}$ with a logarithmic singularity, it is necessary to allow for the logarithmic growth of $w^{j}(\xi)$ as $|\xi| \rightarrow+\infty$. Such solutions of homogeneous problems (21), (22), (23) and (21), (22), (24) are well known (see e.g. [5], [3]). The solutions resemble capacitary potentials in the theory of harmonic functions (see e.g. [12]), belong to the space $H_{\text {loc }}^{2}\left(\overline{\mathbb{R}}_{j}^{2}\right)^{2}$ and admit the following asymptotic representation at infinity:

$$
w^{j}(\xi)=T^{j}(\xi) a^{j}+c^{j}+O\left(\left|\xi^{j}\right|^{-1}\right), \quad\left|\xi^{j}\right| \rightarrow+\infty .
$$

In the case of $j=1, \ldots, I$ (part of the boundary $\partial \mathbb{R}_{j}^{2}$ is clamped) the column $a^{j}$ in (25) can be arbitrary, however,

$$
c_{j}=M^{j} a^{j},
$$

where the symmetric $(2 \times 2)$-matrix $M^{j}$ is called the Wiener elastic capacity matrix for the half-plane clamped along $\left[-l_{j} / 2, l_{j} / 2\right]$. For $j=I+1, \ldots, J$, we have

$$
a^{j}=n\left(P^{j}\right) a_{j}, \quad\left(n^{j}\right)^{\top} c^{j}=m^{j} a_{j},
$$

where the scalars $a_{j}$ and $\left(s^{j}\right)^{\top} c^{j}$ are arbitrary, and $m^{i}$ is a quantity which can be called the Wiener scalar elastic capacity of the interval $\left[-l_{j} / 2, l_{j} / 2\right]$ on the boundary of the half-plane.

REMARK 1. The matrix $M^{j}$ and the scalar $m^{j}$ depend only on the Hooke matrix $A\left(P^{j}\right)$ and on the direction of the normal vector $n^{j}$. Indeed, denoting by $M(l)$ and $m(l)$ these objects for the interval of length $l$, by the contraction $x \mapsto y=l^{-1} x$, in view of representation (15), we obtain

$$
M(l)=M(1)-\mathcal{T}^{j 0} \ln l, \quad m(l)=m(1)-\mathcal{T}^{j 0} n^{j} \ln l .
$$

In the case of isotropic material, $M^{j}$ and $m^{j}$, in addition, are independent of the direction $n^{j}$, and can be determined by using the Kolosov-Muskhelishvili potentials (see [15]).

When we return to the coordinates $x$, by comparison of representations obtained from (25)-(27) and (15),

$$
\begin{aligned}
w^{i}\left(h^{-1}\left(x-P^{i}\right)\right)= & T^{i}\left(x-P^{i}\right) a^{i}+\left(\mathcal{T}^{i 0} \ln h+M^{i}\right) a^{i} \\
& +O\left(h\left|x-P^{i}\right|^{-1}\right), \quad i=1, \ldots, I, \\
w^{j}\left(h^{-1}\left(x-P^{j}\right)\right)= & T^{j}\left(x-P^{j}\right) \nu^{j} a_{j}+\left(\mathcal{T}^{j 0} \nu^{j} \ln h+m^{j}\right) a^{j} \\
& +\left(s^{j}\right)^{\top} c^{j}+O\left(h\left|x-P^{j}\right|^{-1}\right), \quad j=I+1, \ldots, J,
\end{aligned}
$$


with the expansion of the field $v=\mathbf{v}=\mathfrak{v}$ given in (14), the following equalities arise:

$$
\begin{array}{ll}
b^{i}=\left\{\mathcal{T}^{j 0} \ln h+M^{i}\right\} a^{i}, & i=1, \ldots, I, \\
b_{j}=\left\{\mathcal{T}^{j 0} \nu^{j} \ln h+m^{j}\right\} a_{j}, & j=I+1, \ldots, J,
\end{array}
$$

which in vector notation takes the form $\mathbf{b}=\mathbf{S a}$, used already in (16) and indirectly in (18). Thus, the matrix $\mathbf{S}$ is block-diagonal and contains the entries separated in (29) and in (30) by curly braces, respectively $(2 \times 2)$ matrices and scalars. In view of the properties of $\mathcal{T}^{j 0}$ listed after the formula (15), the matrix $\mathbf{S}$ is symmetric and negative definite for sufficiently small $h \in\left(0, h_{0}\right]$.

The relations (29) and (30) are derived by matching the outer expansion $\mathbf{v}=\mathfrak{v}$ with the inner expansions $w^{j}\left(\xi^{j}\right), j=1, \ldots, J$. Therefore, by the Korn inequality (9), (10), proximity to the true solution $u(h, x)$ of the global asymptotic approximation in the energy norm can be established. The global asymptotic approximation is obtained by glueing the expansions in the standard way (cf. [10] and [14], [19]). However, in view of the assumption (12) to model the functional (11) an estimate for the difference $u-\mathbf{v}=u-\mathfrak{v}$ in the $L_{q}(\Omega)^{2}$ norm is required. Such an estimate can be established, taking into account the embedding $H^{1}(\Omega) \subset L_{q}(\Omega)$, by direct evaluation of the $L_{q}(\Omega)$ norms of the remainders in the representations (28).

Theorem 1. If $u$ and $\mathbf{v}=\mathfrak{v}$ are solutions to problems (4)-(8) and (17)-(18), respectively, with the same right-hand side $f \in L_{2}(\Omega)^{2}$, then

$$
\left\|u-\mathbf{v} ; L_{q}(\Omega)\right\| \leq c_{\varkappa} h|\ln h|^{\varkappa+5 / 2}\left\|f ; L_{2}(\Omega)\right\| .
$$

The functional (11) admits the estimate

$$
\left|\mathcal{F}(u ; h)-\int_{\Omega} J(x ; \mathbf{v}(\ln h)) d x\right| \leq C_{\varkappa} \mu_{q}(h)\left\|f ; L_{2}(\Omega)\right\|^{q},
$$

where $\varkappa$ is arbitrary positive, the constants $c_{\varkappa}$ and $C_{\varkappa}$ are independent of $f$ and $h \in\left(0, h_{0}\right]$, and

$$
\mu_{q}(h)= \begin{cases}h|\ln h|^{q(\varkappa+5 / 2)} & \text { for } q \in[1,2], \\ h^{2 / q} & \text { for } q>2 .\end{cases}
$$

Clapeyron's Theorem, stating that the potential energy $=$ the elastic energy minus the work of external forces, takes the form

$$
\mathcal{E}(u ; f)=\frac{1}{2}(A D(\nabla) u, D(\nabla) u)_{\Omega}-(f, u)_{\Omega}=-\frac{1}{2} \int_{\Omega} u^{\top} f d x,
$$


and Theorem 1 can be used to show that the functional (34) evaluated at the solutions to problem (4)-(8) is approximated with error $O\left(\mu_{q}(h)\left\|f ; L_{2}(\Omega)\right\|\right)$ by the energy functionals for the problems (17), (18),

$$
\begin{aligned}
\mathbf{E}(\mathbf{v} ; f) & =\frac{1}{2}(\mathbf{L v}, \mathbf{v})_{\Omega}-(f, \mathbf{v})_{\Omega}, \\
\mathfrak{E}(\mathfrak{v} ; f) & =\frac{1}{2}\left(D(-\nabla)^{\top} A D(\nabla) \mathfrak{v}, \mathfrak{v}\right)_{\Omega}+\frac{1}{2}\left\langle\mathbf{S} \pi^{-} \mathfrak{v}-\pi^{+} \mathfrak{v}, \pi^{-} \mathfrak{v}\right\rangle-(f, \mathfrak{v})_{\Omega} .
\end{aligned}
$$

We refer the reader to [18] for a general approach to modelling of geometrical singularities.

Acknowledgements. The research is supported by INRIA in the framework of a grant from Institut franco-russe A. M. Liapunov d'informatique et de mathématiques appliquées.

\section{References}

[1] G. Allaire, Shape Optimization by the Homogenization Method, Appl. Math. Sci. 146, Springer, New York, 2002.

[2] G. Allaire, F. Jouve and A.-M. Toader, Structural optimization using sensitivity analysis and a level-set method, J. Comput. Phys. 194 (2004), 363-393.

[3] I. I. Argatov, Integral characteristics of rigid inclusions and cavities in the twodimensional theory of elasticity, Prikl. Mat. Mekh. 62 (1998), 283-289 (in Russian); English transl.: J. Appl. Math. Mech. 62 (1998), 263-268.

[4] I. I. Argatov and S. A. Nazarov, Asymptotic solution to the Signorini problem with small parts of the free boundary, Sibirsk. Mat. Zh. 35 (1994), 258-277 (in Russian); English transl.: Siberian Math. J. 35 (1994), 231-249.

[5] V. M. Babich and M. I. Ivanov, Long-wave asymptotics in problems of the scattering of elastic waves, Zap. Nauchn. Sem. Leningrad. Otdel. Mat. Inst. Steklov. (LOMI) 156 (1986), 6-19, 184 (in Russian); English transl.: J. Soviet Math. 50 (1990), $1685-1693$.

[6] M. Bendsoe and O. Sigmund, Topology Optimization, Theory, Methods and Applications, Springer, 2003.

[7] F. A. Berezin and L. D. Faddeev, Remark on the Schrödinger equation with singular potential, Dokl. Akad. Nauk SSSR 137 (1961), 1011-1014 (in Russian); English transl.: Soviet Math. Dokl. 2 (1961), 372-375.

[8] S. Garreau, Ph. Guillaume and M. Masmoudi, The topological asymptotic for PDE systems: the elasticity case, SIAM J. Control Optim. 39 (2001), 1756-1778.

[9] L. Jackowska-Strumiłło, J. Sokołowski, A. Żochowski and A. Henrot, On numerical solution of shape inverse problems, Comput. Optim. Appl. 23 (2002), 231-255.

[10] A. M. Il'in, Matching of Asymptotic Expansions of Solutions of Boundary Value Problems, Transl. Math. Monogr. 102, Amer. Math. Soc., 1992.

[11] - A boundary value problem for the elliptic equation of second order in a domain with a narrow slit. I. The two-dimensional case, Mat. Sb. 99 (1976), 514-537 (in Russian); English transl.: Math. USSR-Sb. 28 (1976), 514-537.

[12] N. S. Landkof, Fundamentals of Modern Potential Theory, Nauka, Moscow, 1966 (in Russian).

[13] D. Leguillon and E. Sánchez-Palencia, Computation of Singular Solutions in Elliptic Problems and Elasticity, Masson, Paris, 1987. 
[14] V. G. Maz'ya, S. A. Nazarov and B. A. Plamenevskii, Asymptotics of Solutions to Elliptic Boundary-Value Problems under a Singular Perturbation of the Domain, Tbilisi Univ., Tbilisi, 1981 (in Russian); Asymptotische Theorie elliptischer Randwertaufgaben in singulär gestörten Gebieten. 1, 2, Akademie-Verlag, Berlin, 1991; Asymptotic Theory of Elliptic Boundary Value Problems in Singularly Perturbed Domains, Vols. 1, 2, Birkhäuser, Basel, 2000.

[15] N. I. Muskhelishvili, Some Basic Problems of the Mathematical Theory of Elasticity, Noordhoff, Leyden, 1975.

[16] S. A. Nazarov, Selfadjoint extensions of the Dirichlet problem operator in weighted function spaces, Mat. Sb. 137 (1988), 224-241; English transl.: Math. USSR-Sb. 65 (1990), 229-247.

[17] - , Two-term asymptotics of solutions of spectral problems with singular perturbations, Mat. Sb. 69 (1991), 291-320; English transl.: Math. USSR-Sb. 69 (1991), 307-340.

[18] —, Asymptotic conditions at points, self adjoint extensions of operators and the method of matched asymptotic expansions, Trudy St.-Petersburg Mat. Obshch. 5 (1996), 112-183 (in Russian); English transl.: Amer. Math. Soc. Transl. 193 (1999), $77-126$.

[19] S. A. Nazarov and B. A. Plamenevsky, Elliptic Problems in Domains with Piecewise Smooth Boundaries, de Gruyter Exp. Math. 13, de Gruyter, 1994.

[20] S. A. Nazarov and J. Sokołowski, Asymptotic analysis of shape functionals, J. Math. Pures Appl. 82 (2003), 125-196.

[21] W. Nowacki, Theory of Elasticity, PWN, Warsaw, 1970 (in Polish); Mir, Moscow, 1975 (in Russian).

[22] B. S. Pavlov, The theory of extension and explicitly soluble models, Uspekhi Mat. Nauk 42 (1987), no. 6, 99-131; English transl.: Soviet Math. Surveys 42 (1987), no. $6,127-168$.

[23] E. Sánchez-Palencia, Forces appliquées à une petite région de la surface d'un corps élastique. Application aux jonctions, C. R. Acad. Sci. Paris Sér. II Méc. Phys. Chim. Sci. Univers Sci. Terre 307 (1988), 689-694.

[24] J. Sokołowski and A. Żochowski, On topological derivative in shape optimization, SIAM J. Control Optim. 37 (1999), 1251-1272.

[25] —, - Optimality conditions for simultaneous topology and shape optimization, ibid. 42 (2003), 1198-1221.

Serguei A. Nazarov

Institute of Mechanical Engineering Problems

Laboratory of Mathematical Methods

Russian Academy of Sciences

V.O. Bol'shoi 61

199178 St. Petersburg, Russia

E-mail:serna@snark.ipme.ru
Jan Sokołowski Institut Elie Cartan Laboratoire de Mathématiques Université Henri Poincaré Nancy I

B.P. 239

54506 Vandœuvre-lès-Nancy Cedex, France E-mail: Jan.Sokolowski@iecn.u-nancy.fr

Received October 6, 2003;

received in final form June 7, 2004 